Instituto Internacional de Investigación y Desarrollo Tecnológico Educativo INDTEC, C.A.

DOI: https://doi.org/10.29394/scientific.issn.2542-2987.2017.2.5.15.283-303

OAI-PMH: http://www.indteca.com/ojs/index.php/Revista Scientific/oai

\title{
Competencias Investigativas Dirigidas a los Profesores de Ingeniería Eléctrica, desde un Modelo de Energía Alternativa
}

Autor: Víctor Hugo Ordóñez Navea

Universidad Fermín Toro, UFT

ordonavea@hotmail.com

Barinas, Venezuela

\section{Resumen}

El propósito del presente trabajo fue plantear competencias investigativas dirigidas a los profesores de ingeniería eléctrica, desde un modelo de energía alternativa y para la explicación del semiconductor en el Programa Nacional de Formación en Electricidad. Algunos autores entre ellos Vidal (2016), Atencio (2014) y Camilo (2012). Señalan posturas tecnológicas de aplicación con los dispositivos electrónicos semiconductores, de esta forma se presenta una fase diagnóstica, apoyada en esta investigación de campo tipo descriptiva sobre: a). Como identificar las necesidades de las energías alternativas, y b). Las competencias investigativas en las energías alternativas del investigador desde un modelo de celda solar, para impulsar e innovar praxis académica e inventiva tecnológica. Así mismo se aplicó una encuesta a un grupo de 15 docentes en el Programa Nacional de Formación en electricidad para diagnosticar la carencia de investigaciones en el campo de las energías alternativas. El procedimiento de análisis de datos se llevó a cabo mediante la estadística descriptiva. Posteriormente se presentan en las conclusiones la necesidad de generar estrategias para estimular y proponer la exploración de energías alternativas al desarrollo de competencias investigativas dirigidas a los profesores de ingeniería eléctrica, desde un modelo de energía alternativa e impulsar la investigación en energías renovables.

Palabras clave: competencias investigativas; ingeniería eléctrica; energía alternativa; praxis académica.

Fecha de Recepción: 16-02-2017

Fecha de Aceptación: 17-03-2017 


\title{
Research Capabilities Directed to all Electric Engineering Teachers, from an Alternative Energy Model
}

\begin{abstract}
The purpose of this work was to contemplate research capabilities directed to all electric engineering teachers from an alternative energy model intro the explanation of a semiconductor in the National Training Program in Electricity. Some authors, such as. Vidal (2016), Atencio (2014) y Camilo (2012) point out to technological applications with semiconductor electrical devices. In this way; a diagnostic phase is presented, held on this field research as a descriptive type about: a) how to identify the necessities of alternative energies, and b) The research competences in the alternatives energies of researcher from a solar cell model, to boost and innovate the academic praxis and technologic ingenuity. Themselves was applied a survey for a group of 15 teachers in the National Program of Formation in electricity to diagnose the deficiencies in the research area of alternatives energies. The process of data analysis was carried out through descriptive statistic. Later the conclusions are presented the need to generate strategies for stimulate and propose exploration of alternatives energies to the development of research competences directed to the teachers of electrical engineering for develop the research competences in the enforcement of the teachers exercise for the electric engineering, from an alternative energy model and boost the technologic research in the renewal energies field.
\end{abstract}

Keywords: investigative competences; electrical engineering; alternative energy; academic praxis.

Date Received: 16-02-2017

Date Acceptance: 17-03-2017 


\section{Introducción}

De forma ecléctica, se busca la raíz epistemológica de cómo se debe aplicar todo este conglomerado de saberes, para incentivar la inventiva tecnológica aplicando los dispositivos electrónicos con el conocer de los semiconductores, contenido principal de la unidad curricular electrónica en la Universidad Politécnica Territorial José Félix Ribas (UPTJFR) del Estado Barinas, núcleo Barinitas dentro de la carrera de ingeniería eléctrica.

En primer lugar, se realizó un diagnóstico utilizando como técnica la encuesta y se aplicó como instrumento un cuestionario, en donde se verificó la falta de interés en la investigación de las energías alternativas, en el cual surgen las interrogantes:

¿Cuáles son las principales necesidades de las competencias investigativas dirigidas a los profesores de ingeniería eléctrica, desde un modelo de energía alternativa?.

¿Cuáles son las competencias investigativas dirigidas a los profesores de ingeniería eléctrica, desde un modelo de energía alternativa?.

De esta manera, la apreciación de los encuentros de los saberes es relevante, ya que el aporte de la unidad curricular electrónica con una inventiva experimental es para estimular la investigación en las energías renovables con la construcción de una celda solar, a partir del transistor de unión bipolar para convertir la energía luminosa del sol, como lo es el fotón en energía eléctrica mediante un dispositivo electrónico semiconductor para la praxis de la unidad curricular electrónica e impulsar competencias investigativas energéticas.

Dentro de este orden de ideas, el propósito de la presente investigación es proponer competencias investigativas dirigidas a los profesores de ingeniería eléctrica, desde un modelo de energía alternativa en la UPTJFR del Estado Barinas, núcleo Barinitas, en la cual se fortalece con la Ley de Uso 
Racional y Eficiente de la Energía (2011), cuyo artículo 22 hace referencia a la educación universitaria en promover la inclusión de contenidos, formación de cátedras, seminarios, talleres o materias que permitan perfeccionar los contenidos de aprovechar las energías renovables para formular propuestas mediante la investigación aplicada en la carrera de ingeniería eléctrica para la educativa de calidad para la calidad de vida.

Asimismo, el artículo 25 expresa que se promoverán programas de investigación científica y tecnológica por parte de las instituciones 0 particulares orientadas a desarrollar nuevas tecnologías y mejorar las existentes en el marco de las energías renovables. De tal manera buscar las competencias energéticas para incentivar la generación de fuentes alternas de energía con el fin de propiciar el uso de fuentes de energías renovables y ambientalmente sostenibles.

\section{Objetivo General}

Proponer las competencias investigativas dirigidas a los profesores de ingeniería eléctrica, desde un modelo de energía alternativa en la Universidad Politécnica Territorial José Félix Ribas del Estado Barinas núcleo Barinitas.

\section{Desarrollo}

\subsection{Antecedentes Investigativos}

Para las investigaciones sobre la situación de las competencias investigativas dirigidas a los profesores de ingeniería eléctrica, desde un modelo de energía alternativa, se localizó como antecedentes los siguientes trabajos, que guardan relación con el estudio, y que es una referencia significativa para la investigación:

Según Vidal (2016), el objetivo fue "proponer lineamientos prácticos que puedan ser utilizados en el mejoramiento de la formación para la investigación en los programas de ingeniería" (pág. 14), con el fin de fortalecer y aumentar 
la investigación desarrollada por los docentes y estudiantes. En donde las estrategias de enseñanza utilizadas adoptan el modelo pedagógico cognitivo contextual de corte constructivista.

En el mismo margen de ideas, Atencio (2014), concibe la relación con los tipos de "competencias investigativas con énfasis en el campo tecnológico" (pág. 18) son abordadas por los estudiantes, en el cual no se desarrolla la inventiva e innovativa, por tal motivo presenta debilidades en la generación de prototipos, progreso industrial y registro de patentes. Por lo tanto, Camilo (2012), plantea que los principales resultados a lograr es el progreso cognoscitivo y de competencia, para obtener un modelo energético económico, sostenible y viable en la carrera de ingeniería eléctrica.

Desde un perfil epistemológico se plantea la búsqueda del conocer y hacer una inventiva experimental, estudios realizados por Piaget (1975), cuando concibe dispositivos experimentales propuestos en poner a poner en evidencia la particularidad del pensamiento de las ciencias exactas, capaz de impulsar competencias investigativas en el campo de energías renovables como es el caso de la energía solar o la energía fotovoltaica. En base al conocer de los dispositivos electrónicos y sus aplicaciones surge esta interrogante, si los dispositivos electrónicos es un semiconductor intrínsecamente dentro de su encapsulado, por qué no lo puedo aplicar como celda fotovoltaica, como lo hago y que me lo impide.

\section{Marco Teórico}

\subsection{Praxis de Semiconductores en la Unidad Curricular Electrónica}

En referencia a la unidad curricular electrónica, que contribuye de forma directa a las energías renovables y específicamente a la energía solar como son las celdas fotovoltaicas las cuales convierten la energía solar en energía eléctrica, nos llevan al conocer de los semiconductores contenido fundamental de la electrónica, en donde la temática de dicha unidad curricular inicia con los 
semiconductores y solo explica el semiconductor como dispositivo electrónico y su aplicación a una red de componentes electrónicos o eléctricos.

\subsection{Las Competencias Investigativas}

Desde la perspectiva del investigador, las competencias desde el enfoque funcional en las dimensiones complejas involucran el proceso de destrezas en los encuentros de saberes con prácticas epistemológicas que cambien las barreras ortodoxas del pensamiento y promuevan reinventar acciones consideradas por el hombre como ser pensante en su entorno en función para la vida de acuerdo a criterios para la investigación.

Según Boylestad y Nashelsky (2009), encontró que permanentemente salen al mercado nuevos dispositivos, donde nuestros ingenieros se encuentran limitados en cuanto a impartir los saberes en referencia a los dispositivos electrónicos, en la cual hay una variedad de avances tecnológicos; De esta manera se debe mantener actualizado sobre las innovaciones y los cambios en un área de investigación o desarrollo. Además, ha llegado a un punto en que solo se utiliza a veces su aplicación y más el conocer del dispositivo, también para suministrar un elemento o tarjeta que permita acoplar al resto del sistema.

En el mismo orden de ideas la electrónica y sus dispositivos según Jasprit (1997), el cual fundamenta la respuesta de esta pregunta:

¿Cómo responde un libro para estudiantes universitarios a los cambios que ocurren en la tecnología de semiconductores?

Con un enfoque espontaneo de la realidad en la praxis académica entre los paradigmas que ocurren en los avances tecnológicos, como es el caso de los semiconductores, en la cual solo se plantea de forma general una minúscula fracción de la física de semiconductores, se ha olvidado profundizar en el tema de estos dispositivos semiconductores, a donde también acarrea el 
estudio de los dispositivos opto electrónicos de semiconductor como lo es el foto receptor, igualmente este se asemeja con la celda solar.

De tal expectativa, ha servido a los ingenieros en electrónica y electricista el conocer y aplicar los dispositivos electrónicos semiconductores a una red o sistema, pero no se ha tenido el atrevimiento para impulsar investigaciones y fortalecer las inventivas tecnológicas en el estudio con el diseño y construcción de dispositivos semiconductores o innovaciones tecnológicas para promover la praxis académica y las investigaciones relevantes a las energías alternativas.

Tradicionalmente, los dispositivos opto electrónicos se han revisado con brevedad en los textos y en los currículos universitarios. Parte de la razón se deriva de un sentimiento de que algo como los dispositivos opto electrónicos, son más mecánica cuántica que dispositivos electrónicos. Mientras que históricamente, los efectos ópticos pueden estar más vinculados a la mecánica cuántica, en realidad el nivel de física necesario no es muy diferente en los dispositivos electrónicos y opto electrónicos.

De la siguiente forma, Fuentes (1995), explica que los "Dispositivos foto receptores. Este grupo incluye los dispositivos que pueden convertir en niveles de iluminación cambios para el valor de algún parámetro eléctrico como (resistencia, tensión, corriente). Cabe destacar estos dispositivos como: foto resistencias, celdas fotovoltaicas, fotodiodos y fototransistores" (pág. 19). Lo que revela, de manera explícita la aplicación de estos dispositivos para generar energía eléctrica no contaminante y responder a las necesidades en nuestro entorno social.

\subsection{Semiconductor}

Según Boylestad y Nashelsky (2009), define de una manera sencilla al semiconductor aclarando el prefijo "semi" es aplicado de forma normal a un rango de nivel entre dos limites, en este mismo orden de ideas se define el 
conductor como cualquier material que deja pasar un flujo de carga eléctrica, debido a esto un semiconductor, es un material creado por ciertos niveles que forman su conductividad el cual tiene su funcionamiento entre los extremos de un aislante o un dieléctrico y un conductor.

De tal manera, el semiconductor es creado con el fin de activar el llamado electrón voltios que es la energía asociada a cada electrón, también la energía se expresa en Joules y la carga de un electrón es $1.6 \times 10 \wedge-19$ Coulombio. Por lo tanto, hay dos formas de alterar la composición de los semiconductores una de ellas es la alteración de la temperatura y otra son los paquetes solares o fotón de luz, de tal manera subir los niveles de temperatura hace que los electrones tengan más energía, ellos con eso se logran colocar nerviosos los cuales comienzan a girar más rápido en su órbita y logran salir o lo que se conoce como un salto de estos electrones.

En otras palabras, según Boylestad y Nashelsky (ob.cit.), presenta el proceso de añadir un material o elemento químico a otro material se conoce como dopaje la cual tiene como habilidad para transformar significativamente las características de este, a través del proceso los materiales semiconductores poseen una estructura cristalina definida donde los átomos se organizan con patrón bien definido por naturaleza periódica, de esta forma un patrón completo se denomina cristal al espacio ordenados de iones (átomos) en forma de bloque y repetido regularmente en tres dimensiones.

Cabe considerar los niveles de energía de los átomos y sus electrones en el exterior, así mismo cambian extraordinariamente al estar solicitado por más de un átomo, la herramienta matemática adecuada para la investigación profunda es la mecánica cuántica de aquí se forma el acoplamiento de capas de electrones exteriores que dan lugar a unas bandas de estados de energía las cuales se encuentran muy cercanas entre sí en el semiconductor, existe la banda de conducción definida como aquella a la que pertenece a los niveles 
de energía en la cual los electrones se encuentran libres para establecer la conducción.

Así mismo, dando utilidad a este dispositivo con la generación de energías fotovoltaicas, se busca verificar el conocimiento esbozado en el libro de semiconductores por Jasprit (1997), sobre el comportamiento de un semiconductor como celda fotovoltaica y no como dispositivo electrónico de semiconductor, como lo plantea Boylestad y Nashelsky (ob.cit.), cabe destacar los diferentes puntos de vista para abordar el estudio del semiconductor en las universidades, de esta forma se logrará indagar con el objeto de estudio y tomar como referencia a las investigaciones planteadas como base de la fortaleza en esta investigación tecnológica.

En vista que poseen materiales $\mathrm{N}$ y $\mathrm{P}$, es el mismo principio de los diodos con la gran diferencia que uno de los materiales se repite para formar los tres pines llamados base, colector y emisor de este dispositivo, el cual no es simétrico. En el presente tema se hace referencia a sus características, funcionamiento y la aplicación del dispositivo electrónico con el fin de aplicarlo a una red o sistema, de esta manera se muestra la composición interna de este dispositivo semiconductor electrónico. Descubierto por Shockey, Brattain y Barden en 1947 (laboratorios Bell).

Desde el enfoque de la unidad curricular electrónica en el tema dos, tiene un contenido complejo a la explicación del Transistor de Unión Bipolar (BJT), de esta manera se realiza una serie de operaciones matemáticas y leyes físicas, para abordar la explicación teórica práctica de las características, funcionamiento y aplicación de este dispositivo electrónico, que contribuye a la funcionalidad real entre los equipos electrónicos que se encuentran en el mercado y en nuestra vida cotidiana, cabe destacar que este dispositivo solo necesita un flujo de corriente por la base mayor o igual a 50 micro. Amperios para entrar en saturación lo que significa que los electrones pululan para lograr que los materiales tipo $\mathrm{N}$ y tipo $\mathrm{P}$ se activen. 
De la siguiente manera, se muestra el transistor como un dispositivo que causa revolución en la electrónica básica y la electrónica de potencia, la cual amplía su fortaleza en la actualidad con los niveles tecnológicos avanzados en la micro y nana electrónica, también se sigue utilizando este dispositivo dentro de los circuitos integrados donde existen miles y millones internamente dentro de los circuitos integrados, debido a su aplicación en la lógica de los sistemas digitales.

Ahora según lo explica Boylestad y Nashelsky (ob.cit.), eliminaremos la polarización base emisor del transistor PNP, asimismo cabe destacar la similitud como se encuentra la situación entre el diodo con polarización inversa y de tal manera se muestra el flujo de los portadores mayoritarios es cero, lo que existe un flujo de portadores minoritarios en el transistor. Cabe destacar de forma explícita que la unión P-N de un BJT se encuentra en polarización inversa, mientras que la otra se encuentra en polarización directa.

La cuestión ahora es, si estos portadores contribuirán de forma directa con la corriente base o si pasarán directo al material tipo P. De este modo existe en el material tipo $\mathrm{N}$ una baja conductividad y si el material tipo $\mathrm{N}$ pertenece al transistor PNP como esta en el centro es muy delgado de forma igual sucede en el NPN pero ahora la condición es ahora que el material tipo $P$ es el que de baja su conductividad y proporciona una información precisa de su funcionamiento interno.

Debe señalarse, que el transistor es otro dispositivo electrónico semiconductor de múltiples aplicaciones, la cual nos lleva aplicar el dispositivo como tal se muestra en la unidad curricular electrónica, cómo unos de los dispositivos semiconductores con mucha relevancia para hacer hincapié en la investigación y su aplicación no solamente como dispositivo semiconductor electrónico, si no también innovar logrando modificar su estructura física y conexiones externas para exponerlo a los fotones de luz. 
Asimismo, generar energía eléctrica mediante los rayos solares; De este modo cambiar la aplicación por el cual fue fabricado y unificando criterios tecnológicos y científicos, con base al modelo del transistor de Ebers Moll, utilizando las leyes físicas de Kirchhoff con la teoría de semiconductores, ya que posee el mismo principio de la celda solar que es un semiconductor.

\subsection{Energías Alternativas.}

Cuando hablamos de energías alternativas son aquellas que se encuentran en nuestro entorno en la naturaleza e inagotable en relación al tiempo de vida del hombre en el planeta tierra y tienen su origen en los procesos ambientales y atmosféricos naturales: el viento, el sol, los cursos de agua, la descomposición de la materia orgánica, el movimiento de las olas en la superficie del mar y océanos, el calor interior de la tierra son fuentes de energías alternativas. En cada caso observamos la presencia de un factor común: el efecto y la acción del sol.

Según Bridgewater, A \& Bridgewater, G (2009), en su libro energías alternativas expresa que "son fuentes de energías no conectadas a la red eléctrica". (pág. 12). Lo que plantea es la situación total independiente de una energía limpia no contaminante, este debe ser nuestro horizonte y aportar lo posible de avanzar en las energías renovables.

Así mismo en este margen de ideas la (ob.cit.), en su libro energías alternativas también propone que "las células fotovoltaicas como la gran promesa a futuro en el campo de las energías alternativas". (pág. 15). De este modo la energía solar para transformarla en energía eléctrica es una alternativa con una proyección viable y en avance de su eficiencia mediante las aportaciones tecnológicas realizadas a nivel internacional.

En la actualidad, la investigación tecnológica se ha convertido en una alternativa para garantizar el acceso de avance y no de complejidad para realizar aportaciones de diseños, la cual muestre de forma creativa y garantice 
el impulso de fortalecer el campo de las energías alternativas, de manera puntual la creatividad e inventiva están asociadas a una necesidad de cambio en la manera de concebir, analizar y resolver los problemas.

En el contenido de semiconductores de la unidad curricular electrónica propone toda la ayuda en el campo de las energías renovables según lo planteado en la Ley de Uso Racional y Eficiente de la Energía (2011), cuyo artículo 6 hace referencia a las definiciones de las energías renovables y las energías alternativas las cuales son aquellas fuentes de energías eléctricas sustituidas por fuentes de energía primaria naturales capaces de regenerarse.

Esta nueva realidad, obliga al docente universitario de las ciencias duras a capacitarse e impulsar las inventivas tecnológicas, en la cual nuestro caso son las energías renovables, Según plantea Gallego, (2004) la idea es enlazar teoría con naturaleza o los fenómenos físicos y químicos que rodean el entorno para investigar de manera experimental y aprovechar su potencial, como es el uso adecuado de la enorme cantidad de energía que se contempla a nuestro alrededor en la naturaleza.

\subsection{Identificar las Necesidades de la Investigación en las Energías} Alternativas.

Para la detección de las necesidades de investigación, inicialmente se revisaron las diferentes propuestas de capacitación de asesores desarrolladas por la coordinación de estudios y el programa de actualización de los docentes de la UPTJFR del Estado Barinas, núcleo Barinitas, logrando observarse que no realizan investigaciones en las energías alternativas, de este tipo como en el diseño de celda solar como estímulo de competencias investigativas dirigidas a los profesores de ingeniería eléctrica, hacia la praxis en las energías alternativas, como el que se propone en el presente trabajo de investigación. 


\section{Metodología}

Este estudio está sustentado en el enfoque cuantitativo y expone una investigación de campo tipo descriptiva que pretende proporcionar una posible solución a una necesidad educativa, en el que confronta la teoría con la realidad en una dirección de invención, mejora o transformación para presentar una inventiva tecnológica en busca de su utilidad. De tal manera según Hernández, Fernández y Baptista (2014), "La investigación descriptiva busca especificar propiedades, características y rasgos importantes de cualquier fenómeno que se analice" (pág. 92).

Adicionalmente, se aplicó la técnica de la encuesta a un grupo de 15 docentes ingenieros electricista e ingenieros en electrónica con un instrumento tipo cuestionario de 22 preguntas para el Programa Nacional de Formación (PNF) en electricidad de la UPTJFR del Estado Barinas, núcleo Barinitas, el cual fue previamente validado con la técnica de juicio de tres (3) expertos dos ingenieros electricista y un licenciado en metodología de la investigación, de tal forma la desviación producida por errores causales, se calculó por medio del tipo de alfa de Cronbach, cuyo resultado arrojo (83,8\%), para la confiabilidad de la muestra y la población. El análisis de datos se realizó mediante la estadística descriptiva, lo que permitió el cálculo de porcentajes para hacer el diagnóstico de la situación presentada.

Esta información recolectada permitió definir claramente las necesidades de la investigación tecnológica en energías alternativas, la cual permitió determinar los siguientes aspectos de relevancia a considerar para el diagnóstico de necesidades en la investigación de energía renovable y de aprendizaje: 
Grafico 1. Investigación en energías renovables de los docentes.

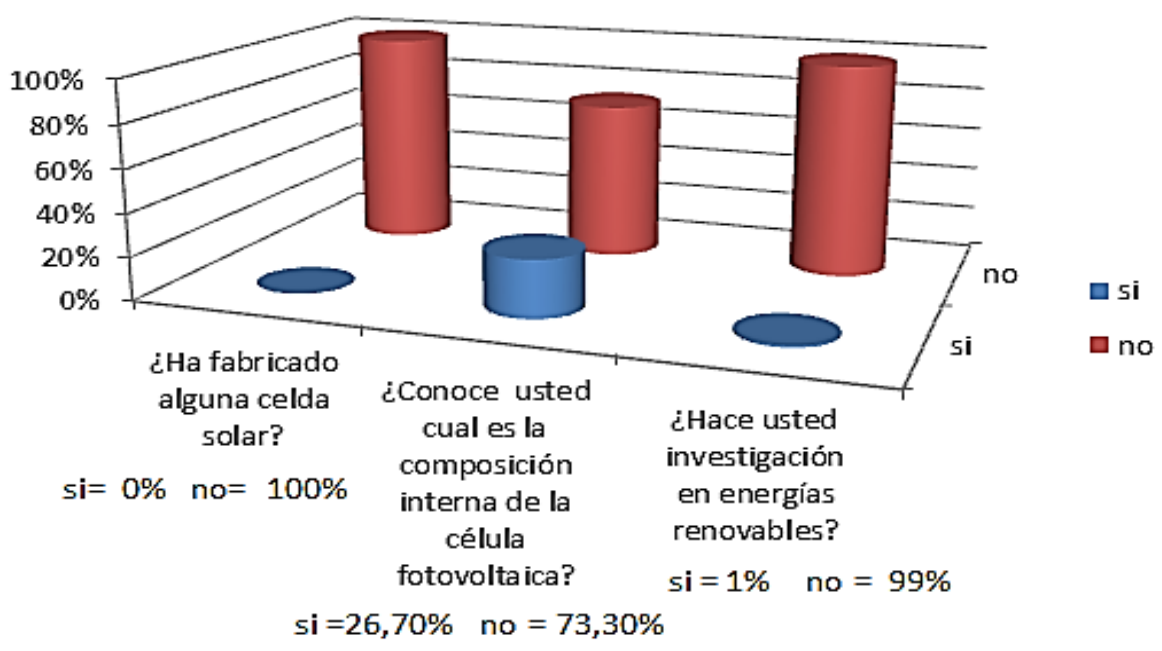

Fuente: El autor (2017)

Es relevante apropiarnos de nuevas tecnologías y crear las nuestras, de esta manera como lo plantea Oppenheimer (2014), en su libro crear o morir expresa que "países latinoamericanos ya tienen grandes reservas de mentes creativas, que son la condición esencial para las sociedades innovadoras, su gran desafío será mejorar la calidad y la inserción en el mundo de su sistema educativo" (pág. 25). En vista de algunas posturas emprendedoras es necesaria la educación de calidad, tomando algunas consideraciones y aporte planteados por la UNESCO (1996) para impulsar la investigación tecnológica en nuestros centros universitarios del conocimiento.

\section{Competencias Investigativas en las Energías Alternativas con un Modelo de Celda Solar para Impulsar la Inventiva Tecnológica.}

Es necesario crear enfoques alternos con los estudiantes y equipos de investigación para promover el dialogo de saberes nacionales e internacionales con el objeto de impulsar congresos y eventos, en la cual se encuentre enmarcada de manera dimensional las inventivas tecnológicas, con 
la acción de aplicar las relaciones entre una reorganización del mercado del mundo productivo empresarial y el estado, este dialogo debe coexistir dentro del contexto dinámico globalizador, frente a la fuerza en movimiento de un nuevo modelo que emerge de los entornos sociales.

Cabe destacar de forma estratégica la clave de las competencias investigativas para la calidad de vida en un ambiente sustentable, la cual la UPTJFR del estado Barinas, núcleo Barinitas no se propaga el dinamismo creativo e innovador con indicadores y controles de las líneas investigativas en el campo de las energías renovables con invectivas tecnológicas, incluso deben ser cónsonas con los problemas del entorno social para producir la calidad educativa para la vida.

El acelerado desarrollo del conocimiento a través de las investigaciones tecnologías, representa actualmente un impacto educativo importante en los últimos tiempos. Como lo es la energía solar para producir energía eléctrica limpia no contaminante, de tal manera se hace énfasis en la inventiva tecnológica de una celda solar capaz de generar energía eléctrica en base a los dispositivos electrónicos semiconductores y los fotones recibidos por el astro mayor que es el sol una fuente inagotable de energía.

Con esta iniciativa de promover e incitar a la búsqueda tecnológica aplicando los saberes entre las interacciones de los docentes y estudiantes, en la cual lógicamente tienen el compromiso de estar actualizándose, de tal modo apoyando diseños creativos que analicen las diferentes variables de un sistema de energía fotovoltaica, con la integración de las unidades curriculares a los proyectos energéticos alternativos que aporten respuesta a las necesidades del entorno. Es necesario entonces reorientar y coordinar los esfuerzos con el objeto de insertar la exploración en energías alternativas en la dinámica educativa como un instrumento para el logro de los más importantes fines de las instituciones universitarias. 
De aquí, la importancia de diseñar la celda solar para el estímulo de competencias investigativas dirigidas a los profesores de ingeniería eléctrica, hacia la praxis en las energías alternativas en la UPTJFR del Estado Barinas núcleo, Barinitas, el cual pretende desarrollar con los docentes universitarios las habilidades necesarias en el manejo de promover y estimular la inventiva tecnológica en las energías renovables para el PNF en Electricidad con la aportación de un modelo experimental de una celda solar en el desarrollo y aplicación de las unidades curriculares, de este modo generar nuevas formas de relación y conocimiento entre los estudiantes, estudiantes y docentes, y entre docentes.

A partir de la investigación realizada al enfoque situacional del problema de estudio, se presenta una serie de competencias adecuadas al cumplimiento del ejercicio docente en el cómo se:

- Planifica y modela la creación en el campo de energías renovables para promover la inventiva tecnológica en los estudiantes.

- Desarrolla indicadores para la investigación en energías renovables.

- Usa el aprendizaje significativo de la aplicación de los dispositivos electrónicos semiconductores para motivar la investigación.

- Aplica la información de las variables en el diseño de la celda solar dentro del campo de las energías alternativas.

- Establece una interpretación de las normas y un control de su cumplimiento de tal manera que se estimule y fomente la capacidad de inventiva a través del perfeccionamiento de los distintos conceptos en energías renovables.

- Usa el dispositivo semiconductor en aplicación como foto receptor para transformar la energía solar en energía eléctrica y de esta manera impulsar la inventiva tecnológica. 
- Promueve y estimula la inventiva tecnológica que responda a necesidades socio productivas y sustentables derivadas del saber hacer en las energías alternativas.

- Desarrolla nuevos avances tecnológicos para generar energía renovable.

- Construye indicadores para fomentar la inventiva tecnológica en el marco de las energías alternativas.

- Promueve y estimula la investigación aplicada en energías solar.

- Promueve y estimula la construcción de una celda solar experimental.

\section{Conclusiones}

En la educación, hoy en día existe una renovación y transformación de la manera de adquirir conocimiento y de la forma de investigar, profundizar la información para lograr generar el conocer con la aplicación en el área de la inventiva tecnológica en busca de la practica experimental del conocer a través de las diversas vías de los encuentros de los saberes, cabe destacar la búsqueda de estrategias para estimular y proponer la exploración de energías alternativas, la cual constituye un instrumento muy valioso para llegar a profundizar en el campo de las energías renovables.

Asunto por el cual, se hace necesario que los profesores se actualicen en el área tecnológica, de ahí nace esta investigación, que en atención a los objetivos planteados al comienzo, se concluye, que se logró diagnosticar la necesidad de incitar la investigación en energía solar en los docentes y los estudiantes del PNF en electricidad de la UPTJFR del Estado Barinas, núcleo Barinitas, en la cual logren asumir el modelo de una celda solar como inventiva tecnológica para proyectar estratégicamente el campo de las energías renovables.

De esta manera, se alcanzó a determinar que gran parte de los docentes y los estudiantes requerían insertarse para el campo de la inventiva 
tecnológica de energías renovables, ya que presentaban desconocimiento de muchos elementos importantes para la interacción del conocer y aplicar conocimientos que logren aportar en las energías alternativas, en la cual no se ha hecho investigación experimental en las energías renovables en forma directa para generar energía eléctrica no contaminante.

Así mismo, en base a este diagnóstico se elaboró una propuesta para las competencias investigativas dirigidas a los profesores de ingeniería eléctrica, desde un modelo de energía alternativa en la Universidad Politécnica Territorial José Félix Ribas del Estado Barinas, núcleo Barinitas, en la cual impulsará la planificación estratégica para el fortalecimiento de las investigaciones en las energías renovables y en el cumplimiento académico de las creaciones intelectuales.

\section{Recomendaciones}

El uso de un sistema para la planificación de las líneas del campo de investigación en energías renovables, facilita enormemente el diseño con el montaje de una celda solar para estimular la investigación en las energías alternativas.

Asunto por el cual, las competencias investigativas dirigidas a los profesores de ingeniería eléctrica, desde un modelo de energía alternativa con la ayuda de la unidad curricular electrónica bajo el contenido de semiconductores, en donde constituye una excelente oportunidad para conocer una importante estrategia como aprendizaje significativo para el desarrollo de los encuentros de saberes en el aula de clase, como apoyo o complemento para la inventiva tecnológica.

Por tal motivo, es necesario el desarrollo de competencias en el diseño, de planificación y desarrollo de actividades creativas, que sean soportadas o mediadas por las investigaciones en energías renovables o centros de investigaciones energéticas, en donde el docente deberá capacitarse en todos 
los aspectos relativos a la investigación tecnológica del proceso de energías alternativas pertinentes al campo radial de acción de los saberes.

\section{Referencias}

Asamblea Nacional de la República Bolivariana de Venezuela, (2011). Ley de

Uso Racional y Eficiente de la Energía. Gaceta Oficial № 39.823 , diciembre 19, 2011. Venezuela.

Atencio, E. (2014). Competencias Investigativas con énfasis en el Campo Tecnológico en estudiantes universitarios. Realizada en la Universidad Rafael Belloso Chacín en Venezuela. Revista electrónica de humanidades, educación y comunicación social REDHECS, Edición No 18 - Año 9, 143-161.

Boylestad, R. \& Nashelsky, L. (10ra Ed.). (2009). Electrónica: Teoría de Circuitos y Dispositivos Electrónicos. Editorial Prentice Hall. México. Bridgewater, A. \& Bridgewater, G. (1ra Ed.). (2009). Energías Alternativas. Editorial Paraninfo, S.A. Madrid España.

Camilo, W. (2012). Enfoque Curricular para el Desarrollo de Competencias Profesionales, para la Construcción, Explotación y Comercialización de Equipos de Energías Renovables. Tesis del doctorado en educación en energías renovables de la Universidad Alas Peruanas. Lima, Perú. Recuperado de:

http://myslide.es/documents/tesis-doctorado-en-educacion-energiasrenovables-camilo-uap-2009-2012.html

Fuentes, O. (1995). Apuntes de Electrónica II. Universidad de Los Andes. Escuela de Eléctrica, Estado Mérida Venezuela.

Gallego, R. (2004). Un Concepto Epistemológico de Modelo para la Didáctica de las Ciencias Experimentales. Revista Electrónica de Enseñanza de las Ciencias. Volumen 3, número 3, pp. 1-19. España. 
Hernández, R. Fernández, C y Baptista, M. (6ta Ed.). (2014). Metodología de la Investigación. Editorial. McGraw-Hill /interamericana editores, S.A. De C.V. México.

Jasprit, S. (1ra. Ed.). (1997). Dispositivos Semiconductores. Editorial McGraw Hill/Interamericana editores, S.A. De C.V. México.

Oppenheimer, A. (2014). ¡Crear o morir! La esperanza de Latinoamérica y las Cinco Claves de la Innovación. Editorial. Penguin Random House Grupo México.

Piaget, J. (1975). Introducción a la Epistemología Genética. El Pensamiento Matemático. Biblioteca de Psicología Evolutiva. Paidós, Buenos Aires.

UNESCO (1996). Ciencia y Tecnología en América Latina y el Caribe. Cooperación para el desarrollo, UNESCO, Montevideo.

Vidal, C. (2016). Formación para la Investigación en Programas de Ingeniería. Tesis doctoral en ciencias de la educación realizada en la Universidad Rafael Belloso Chacín. Maracaibo, Venezuela. 


\section{Víctor Hugo Ordóñez Navea}

e-mail: ordonavea@hotmail.com

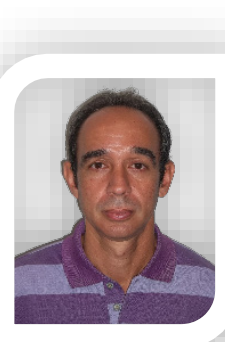

Lugar de Nacimiento San Rafael de él Mojan Estado Zulia, Venezuela. Residenciado en el Estado Barinas Municipio Bolívar "Barinitas", edad: 43 años. Profesor de la Universidad Politécnica Territorial José Félix Ribas del estado Barinas del "PNFE", en el escalafón de Agregado. Egresado de la URBE (Universidad Rafael Belloso Chacín) recibiendo el título de Ing. En Electrónica. ULA (2014). Ponente y tecnólogo Primer Congreso Internacional de Simulación aplicada a la Ingeniería. Egresado de la UFT (Universidad Fermín Toro), recibiendo título de M.Sc. Educación Universitaria. Doctorando en Ciencias de la Educación. 